\title{
Follow Up The Children With Ocular Lesion Induced by Two Common Intrauterine Infections(Cytomegalovirus,Toxoplasmosis): A Cohort Study in Tehran,Iran
}

mohammad Vafaee-Shahi

Iran university of medical sciences

samileh noorbakhsh ( $\sim$ samileh_noorbakhsh@yahoo.com )

Samileh Noorbakhsh, MD ProfessIUMS; Iran univeriaty of medical science ious Diseases, 4th floor Rasul Akram Hospital, Niayesh Street, Satarkhan Avenue, Tehran, 14455 Islamic Republic of Iran. Tel-fax:09821-66516049 https://orcid.org/0000-0002-1656-2388

leila Tahernia

Tehran University of Medical Sciences

sarvenaz ashouri

Iran university of medical sciences

\section{Research Article}

Keywords: cytomegalovirus, ocular disorders, T. Gondii, intrauterine infections, infants.

Posted Date: June 18th, 2020

DOI: https://doi.org/10.21203/rs.3.rs-34738/v1

License: (1) (i) This work is licensed under a Creative Commons Attribution 4.0 International License.

Read Full License 


\section{Abstract}

Background: Intrauterine infections lead to involvement of various organs in fetus, including the eye. The aim of this study was to determine the frequency and clinical response of ocular lesions to specific drugs in infants with congenital Toxoplasma and cytomegalovirus infections.

Methods: This historical cohort study was performed in the pediatric and ophthalmology department of Hazrat Rasool Akram Hospital in Tehran from October 2011 to November 2017. Patients included 78 infants with intrauterine infection (proven) and ocular involvement (cataracts, glaucoma, and retinitis). Infants who did not undergo additional and diagnostic tests, eye examinations, follow-up and did not receive effective treatment were excluded from the study. Three patients expired during the study. Finally, 37 patients (including 25 patients with cytomegalovirus and 12 patients with toxoplasma) were included to assess and the clinical response to ocular lesions was evaluated for one year.

Results: Of 12 cases with toxoplasmosis, 5 patients received complete treatment and 4 patients had appropriate clinical responses. Of 25 patients with CMV, 18 patients received complete treatment and 9 patients had appropriate clinical responses.

Conclusion: Successful treatment will be obtained in near $80 \%$ of ocular toxoplasmosis,so anti toxoplasma treatment is recommended in all confirmed cases .About $50 \%$ of CMV infected cases (with hearing loss, ocular involvement ) might respond well to antiviral therapy, We recommend anti CMV treatment in any age ( $<2$ years old) but it is not indicated in CMV infected cases with severe brain involvement.

\section{Introduction}

Congenital or intrauterine infections are significantly important in all countries around the world in terms of prenatal control and postpartum diagnosis. Despite widespread use of vaccines to control common childhood infections, even in developed countries, infections such as cytomegalovirus, herpes, and other respiratory viruses or parasites such as toxoplasmosis cannot be prevented and may result in intrauterine infection syndrome in infants. The prevalence of cytomegalovirus in the world is between 40 and 100\%. Toxoplasma is an important cause of intrauterine infection in neonates.

The prevalence of anti-toxoplasma antibodies varies in women of childbearing age in every country and even in different parts of a country(1-4). The classic characteristics of congenital infections includes fever, hydrocephalus, microcephalus, hepatosplenomegaly, icter, seizure, chorioretinitis, cerebral calcification, and increased protein and mononeuclear poleocytosis in cerebrospinal fluid(5). It seems that the probability of developing congenital toxoplasmosis is much higher in Iran compared to the United States, about more than 60 to 80 times(6,7).

According to the studies conducted in Iran similar to other countries, cytomegalovirus is one of the most common intrauterine infections. The previous studies in Rasool Akram hospital with the aim of neonatal 
screening had shown that cytomegalovirus was more common but had milder symptoms compared to other causes of TORCH(8). In Iran, toxoplasmosis screening has not yet been performed, while the incidence of toxoplasmosis is so high to be screened. Prenatal screening can help identify mothers who are susceptible to infection. Screening for antibodies enables early prevention of toxoplasmosis infection in which dietary habits and hygiene practices are clearly identified as risk factors(9). Recent studies have shown that frequency of immunoglobulin $(\mathrm{Ig}) \mathrm{G}$ and $\mathrm{M}$ against toxoplasmosis were $44.1 \%$ and $1 \%$ respectively which was less than frequency in mothers. In all cases, the polymerase chain reaction (PCR) of the cerebrospinal fluid was negative. The most common symptoms of congenital infection were ocular $(50 \%)$ and cerebral (50\%) symptoms(10).

The purpose of this study was to determine the frequency and rate of response to treatment in ocular lesions in children with various types of intrauterine infections.

\section{Methods}

The current historical cohort study was performed on all children aged up to 2 years referred to the clinic of ophthalmology department of the Rasool akram Hospital in Tehran to diagnose and treat important ophthalmic problems from October 2011 to November 2017.

This study has adhered to the principles of Helsinki and has been approved by the ethics committee of the Infectious Diseases Research Center of Children affiliated with Iran University of Medical Sciences with code of 9321165001 .Data from all individuals was also retained by the researchers. The all taken measures were after receiving informed consent from parents and all were completely necessary and very helpful for the diagnosis and treatment of referred patients.

Cases definition: Children with ocular lesions caused by intrauterine CMV and toxoplasmosis infections were included in this study.

All patients with eye lesions were followed regularly. Follow up sessions for at least a whole year were recorded in the file, and the results of ophthalmology examinations were recorded and finally evaluated. After this stage, all patients were referred to pediatrician in order to filling out the questionnaire, conducting additional evaluations according to the history and clinical examination, performing additional tests (if necessary) and finally study variables. The possible causes of ophthalmic lesions were identified. After diagnosing the site of involvement inside of the eye in each patient (retinitis, glaucoma, cataract) for final diagnosis, determining the cause of ophthalmic lesions, examining other systemic problems in the child, all were referred to a pediatric clinic. Ultimately, in some patients intrauterine infection was diagnosed.

Exclusion criteria: Patients who did not reach the final diagnosis, despite the available examinations, did not undergo additional and diagnostic tests and were excluded did from our study. Those who acted contrary to the ophthalmologist prescribed instructions for treatment or follow-up were excluded as well from the study. 
Statistic: the frequency for the qualitative variables was expressed based on the mean and standard deviation and for the qualitative variables as the percentage. Comparison between quantitative variables was performed by Student's t-test and comparisons between qualitative variables were also performed using Chi-square test or Fisher's exact test. SPSS software, version 18 (IBM SPSS, Armonk, NY, USA) was used to analyze the data statistics.Levels less than 0.05 were considered significant.

\section{Results}

Totally, between 78 cases of patients with the diagnosis of Intra uterine infection , 37 of them had ophthalmic lesions. Of total cases, 25patients were infected with CMV and 12 were infected with toxoplasmosis. Three patients expired meanwhile. Other patients did not receive antiviral treatment due to parental dissatisfaction, the severity of brain involvement and parental frustration with complete recovery. Due to the mentioned causes, medical treatment were limited to supportive measures such as cerebral shunting, treatment of heart problems and cochlear implants.

Thirty-seven children were treated and examined for ophthalmic lesions resulted from intrauterine infection. Thirteen cases had specific treatment responses and 24 children did not respond to treatment. Of 12 children with ocular toxoplasmosis, only five patients continued their treatments, with four $(80 \%)$ appropriate clinical response, but only one patient (20\%) did not improve significantly despite treatment. Of 25 infants with cerebral involvement caused by congenital CMV infection, only 18 cases continued treatment. Totally 9 cases showed appropriate clinical responses and remaining 9 patients did not improve significantly despite antiviral therapy. One case did not follow examining sessions after treatment. The lack of response to CMV treatment was mainly observed in patients with severe brain lesions from birth. The best response to treatment was reported in patients with hearing loss, ocular involvement and no brain involvement. Comparison between patients' clinical response to antiviral drugs based on sex and other variables could not be concluded due to the small number of cases. The ophthalmic involvement was not different between two groups of with and without clinical response $(P=$ $0.3)$. The mean age of patients who had a good clinical response did not differ significantly from the unresponsive group. ( $4 \pm 1.5$ compared to $4.2 \pm 2.5$ months $)(P=0.7)$.

\section{Discussion}

In present study 25 patients with ocular lesion were infected with CMV and 12 were infected with toxoplasmosis. The ocular lesions responded well to specific treatment in $80 \%$ of toxoplasma cases and $50 \%$ of cytomegalovirus patients.

The unresponsive of CMV infection to antiviral therapy was generally observed in patients with severe brain damage at birth (due to severity and involvement in the early months of fetal period). The best response to treatment was detected in patients with hearing loss and ophthalmic lesions without cerebral involvement. The clinical response of CMV to specific drugs was not dependent on age of them. On the 
other hand, congenital CMV infection has been one of the most common causes in infants suspected of having intrauterine infections[11].

In a CMV study in Isfahan they reported that in 1617 neonates only 8 cases at birth (49\%) were positive in urine CMV-PCR (Polymerase chain reaction) that were mostly immature. In 3 patients, the clinical symptoms were temporary and resolved. In one case, a slight hearing loss was reported in the next follow-up (12).

The first epidemiological study of toxoplasma in pregnant women in Tehran showed that totally $34.3 \%$ of patients had previous immunity and 7\% with recent antibody ( $\operatorname{lgM})$, which did not differ from the prevalence of toxoplasma infection in voluntary blood donors in Iran.

About $35 \%$ of blood donors (IgG and IgM antibodies were $31.4 \%$ and $2.74 \%$ positive, respectively(13). In a recent meta-analysis study in Iran, it was shown that pregnant women accidentally had $41 \%$ antibodies against toxoplasma. IgG was $34 \%$ and IgM $4 \%$. This varied in different provinces. The highest safety was $53 \%$ in the southern parts and the lowest in the eastern regions with $33 \%$ (9). Congenital toxoplasmosis infections caused by maternal infection during pregnancy have been identified as a common cause of intrauterine infection. In another perspective study, congenital toxoplasmosis in 270 infants was evaluated on umbilical cord blood at birth. According to this study, IgG was positive in $44 \%$ and IgM in $1.5 \%$ of neonates. Only $50 \%$ of IgM positive cases had ocular symptoms and $50 \%$ had cerebral symptoms (10).

\section{Conclusion}

Conclusion: Successful treatment will be obtained in near $80 \%$ of ocular toxoplasmosis,so anti toxoplasma treatment is recommended in all confirmed cases .About $50 \%$ of CMV infected cases (with hearing loss, ocular involvement ) might respond well to antiviral therapy, We recommend anti CMV treatment in any age ( $<2$ years old) but it is not indicated in CMV infected cases with severe brain involvement.

\section{Declarations}

\section{Ethics approval and consent to participate}

This study was accredited by Ethical Committee of Iran University of Medical Sciences. Helsinki Declaration was respected across the study and the informed consent form was signed by the parents.

\section{Competing interests}

The authors declare no conflict of interest in preparing this study.

\section{Funding}


This study received no specific grant from any funding agency in the public, institutions or not-for-profit sectors.

All authors confirm Consent for publication

All authors confirm Availability of data and materials

\section{Authors' contributions}

NS designed and supervised the study, writing the initial report.

VM visited and interpreted the patients data; AS and RA analyzed data

TL were major contributors in rewriting and English editing the manuscript.

SA methodology and Analysis $d$

All of authors read and approved the final manuscript.

\section{Acknowledgment}

This paper is based on the result of a dissertation entitled "Determining the frequency and response rate of ophthalmic lesions in children with various types of intrauterine infections (cytomegalovirus and toxoplasmosis)" in 2017 and code of 3081 has been implemented with the support of the Infectious Diseases Research Center of Iran University of Medical Sciences.

\section{References}

1. Kenneson A, Cannon MJ. Review and meta-analysis of the epidemiology of congenital cytomegalovirus (CMV) infection. Rev Med Virol 2007;17(4):253-76.

2. Dollard SC, Grosse SD, Ross DS. New estimates of the prevalence of neurological and sensory sequelae and mortality associated with congenital cytomegalovirus infection. Rev Med Virol 2007;17(5):355-63.

3. Yamamoto AY, Mussi-Pinhata MM, Isaac Mde L, Amaral FR, Carvalheiro CG, Aragon DC, et al. Congenital cytomegalovirus infection as a cause of sensorineural hearing loss in a highly immune population. Pediatr Infect Dis J 2011;30(12):1043-6.

4. James SH, Kimberlin DW. Advances in the prevention and treatment of congenital cytomegalovirus infection. Curr Opin Pediatr 2016;28(1):81-5.

5. Barbi M, Binda S, Caroppo S, Ambrosetti U, Corbetta C, Sergi P. A wider role for congenital cytomegalovirus infection in sensorineural hearing loss. Pediatr Infect Dis J 2003;22(1):39-42.

6. Thalib L, Gras L, Romand S, Prusa A, Bessieres MH, Petersen E, et al. Prediction of congenital toxoplasmosis by polymerase chain reaction analysis of amniotic fluid. BJOG 2005;112(5):567-74. 
7. Noorbakhsh S, Farhadi M, Tabatabaei A, Mohamadi S, Jomeh E. Infection in childhood sensory hearing loss. Saudi Med J 2008;29(10):1470-4.

8. Naing ZW, Scott GM, Shand A, Hamilton ST, van Zuylen WJ, Basha J, et al. Congenital cytomegalovirus infection in pregnancy: a review of prevalence, clinical features, diagnosis and prevention. Aust N Z J Obstet Gynaecol 2016;56(1):9-18.

9. Rossi P. Diagnostic kits in parasitology: which controls? Parassitologia 2004;46(1-2):145-9.

10. Noorbakhsh S, Kalani M, Ali Akbari A. prevalence of congenital toxoplasmosis in newborns in two educational hospitals in Tehran Iran. Arch Dis Childhood 2014;99:A323

11. Rhead WJ, Irons M. The call from the newborn screening laboratory: frustration in the afternoon. Pediatr Clin North Am 2004;51(3):803-18, xii.

12. Ghaemian M, Maraghi SH, Saki J, Pedram M. Determination of antibodies (IgG, IgM) against toxoplasma gondii in patients with cancer. Iran J Parasitol 2007;4(2):1-6.

13. Foroutan-Rad M, Khademvatan S, Majidiani H, Aryamand S, Rahim F, Malehi AS. Seroprevalence of Toxoplasma gondii in the Iranian pregnant women: A systematic review and meta-analysis. Acta Trop 2016;158:160-169.

\section{Figures}

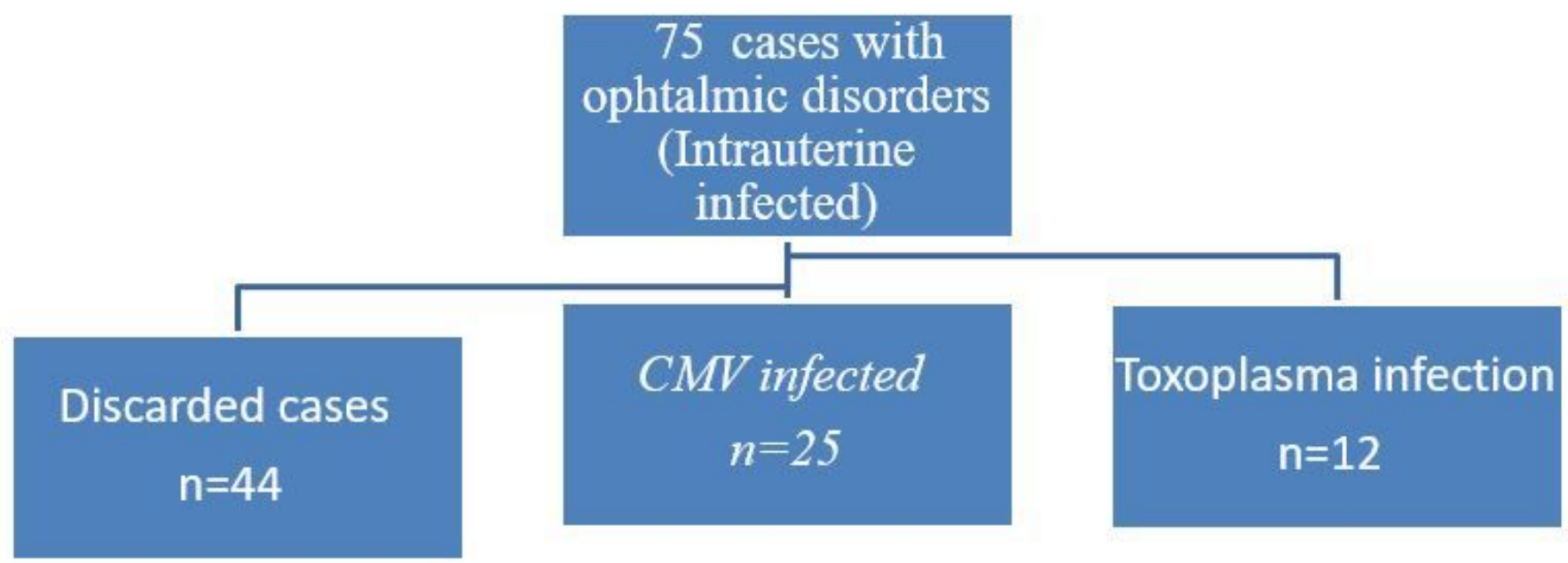

\section{Figure 1}

selection of Cases with ocular disorders due to intrauterine infection

\section{Supplementary Files}

This is a list of supplementary files associated with this preprint. Click to download. 
- personalcover.docx 